doi:10.13108/2020-12-3-50

\title{
ON APPROACH FOR STUDYING STOCHASTIC LEONTIEF TYPE EQUATIONS WITH IMPULSE ACTIONS
}

\author{
E.YU. MASHKOV
}

\begin{abstract}
We study a system of Itô stochastic differential equations having a degenerating constant linear operator in the left hand side. The right hand side of the system contains a constant linear operator and a deterministic term depending on the time only as well as impulse actions. We assume that the diffusion coefficient of this system is described by a square matrix depending on time only. These systems of equations arise in many applications. The system we study can be reduced to a canonical form by applying a transformation of a regular matrix pencil to a generalized real Schur form. The study of the obtained canonical equations requires considering the derivatives of rather higher orders for free terms including the Wiener process. Because of this, in order to differentiate the Wiener process, we apply the Nelson mean derivatives for random processes and this allows us to avoid using the theory of generalized functions. As a result we obtain analytic formulae for solutions of equations in terms of mean derivatives for random processes.
\end{abstract}

Keywords: mean derivative, current velocity, Wiener process, stochastic equations of Leontief type

Mathematics Subject Classification: $60 \mathrm{H} 30,60 \mathrm{H} 10$

\section{INTRODUCTION}

We consider a degenerate Itô stochastic differential equations of form

$$
d \tilde{A} \xi(t)=\tilde{B} \xi(t) d t+f(t) d t+d S \zeta(t)+P(t) d w(t), \quad 0 \leqslant t \leqslant T,
$$

where $\tilde{B}+\lambda \tilde{A}$ is a regular pencil of real constant matrices of size $n \times n$, the matrix $\tilde{A}$ is degenerate, $\xi(t)$ is an unknown random process, $w(t)$ is a Wiener process in $\mathbb{R}^{n}, P(t)$ is a sufficiently smooth matrix of size $n \times n, f(t)$ is a sufficiently smooth $n$-dimensional vector function, $\zeta(t)$ is an $n$-dimensional jump process, $S$ is an $n \times n$-matrix. In literature, the following names are used for these systems: algebraic-differential, descriptor, Leontief type systems. These equations arose in works by L.A. Vlasenko, Yu.G. Lysenko, S.L. Lyashko, A.G. Rutkas [1], [2] in mathematical modelling of enterprise corporation dynamics under an investing. In works by O. Schein, G. Denk [3], T. Sickenberger, R. Winkler [4], [5] such system arose in mathematical modeling of oscillations and electric chains. In works by A.L. Shestakov, G.A. Sviridyuk [6], Leontief type systems were used to study a dynamical distortion of signals in radio devices. We also mention a work by A.A. Belov, A.P. Kurdyukov [7], in which numerous applications of Leontief type equations were described.

A starting point for our paper was work [8], in which this equation was studied by means of Weierstrass canonical form for a regular matrix pencil. Since in the general case we can not stably calculate the Weierstrass form, see [9], this motivates the studying of the system by

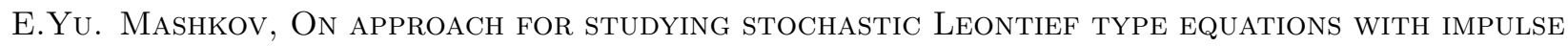
ACTIONS.

(c) Mashkov E.Yu. 2020.

The work is supported by Russian Foundation for Basic Researches (grant no. 18-01-00048).

Submitted April 17, 2020. 
applying the generalized Schur form for regular pencils. As it is known, see [9], the Schur form is calculated in a stable way.

A specific nature of Leontief type equations suggests to consider higher order derivatives of the right hand side, including that for the Wiener process. As it is known, see, for instance, [10], the derivatives of the Wiener process are well-defined only in the sense of generalized functions, which are extremely troublesome for applications in studying of particular equations. This fact makes very difficult a direct studying of our equation.

As in [8], [11, an approach proposed in the present work for studying this equation is based on employing the theory of Nelson mean derivatives of random processes; these derivatives are described with involving no generalized functions. Namely, we use symmetric mean derivatives (current velocities) of the Wiener process. According a general idea of the theory of Nelson mean derivatives, the current velocities are natural analogues of the physical velocity of deterministic processes. As a result, for the studied equation, we obtain formulae for solutions in terms of symmetric mean derivatives of random processes with a physical meaning.

\section{MEAN DERIVATIVES}

We consider a stochastic process $\xi(t)$ in $\mathbb{R}^{n}, t \in[0, l]$, defined on some probabilistic space $(\Omega, F, P)$ such that $\xi(t)$ is a $L_{1}$-random variable for all $t$. It is known that each such process generates a family of $\sigma$-subalgebras of $\sigma$-algebra $F$ a "true" $N_{t}^{\xi}$, which is assumed to be complete, that is, to be completed by all sets of zero probability.

For the sake of convenience we denote $E_{t}^{\xi}$ by a conditional expectation $E\left(\cdot \mid N_{t}^{\xi}\right)$ with respect to the "true" $N_{t}^{\xi}$ for $\xi(t)$. The usual mathematical expectation is denoted by $E$.

Generally speaking, almost all sample trajectories of the process $\xi(t)$ are non-differentiable and its derivatives exist only in the sense of generalized functions. In order to avoid usage of generalized functions, according Nelson [12], [13], [14], we give the following definition:

Definition 2.1 ([10]). (i) A right mean derivative $D \xi(t)$ of a process $\xi(t)$ at time $t$ is a $L_{1}$-random variable of form

$$
D \xi(t)=\lim _{\triangle t \rightarrow+0} E_{t}^{\xi}\left(\frac{\xi(t+\triangle t)-\xi(t)}{\triangle t}\right),
$$

where the limit is assumed to exist in $L_{1}(\Omega, F, P)$ and $\triangle t \rightarrow+0$ means that $\triangle t$ tends to zero 0 and $\triangle t>0$.

(ii) A left mean derivative $D_{*} \xi(t)$ of a process $\xi(t)$ at time $t$ is a $L_{1}$-random variable

$$
D_{*} \xi(t)=\lim _{\triangle t \rightarrow+0} E_{t}^{\xi}\left(\frac{\xi(t)-\xi(t-\triangle t)}{\triangle t}\right),
$$

where, as in (i), the limit is assumed to exist in $L_{1}(\Omega, F, P)$ and $\triangle t \rightarrow+0$ means that $\triangle t$ tends to 0 and $\triangle t>0$.

It should be stressed that generally speaking, $D \xi(t) \neq D_{*} \xi(t)$, but, if, for instance, $\xi(t)$ almost sure has smooth sample paths, these derivatives obviously coincide.

It follows from the properties of the conditional mathematical expectation, see [15], that $D \xi(t)$ and $D_{*} \xi(t)$ can be represented as superposition of $\xi(t)$ and Borel vector fields (regressions)

$$
\begin{aligned}
& Y^{0}(t, x)=\lim _{\triangle t \rightarrow+0} E_{t}^{\xi}\left(\frac{\xi(t+\triangle t)-\xi(t)}{\triangle t} \mid \xi(t)=x\right) \\
& Y_{*}^{0}(t, x)=\lim _{\triangle t \rightarrow+0} E_{t}^{\xi}\left(\frac{\xi(t)-\xi(t-\triangle t)}{\triangle t} \mid \xi(t)=x\right)
\end{aligned}
$$

on $\mathbb{R}^{n}$, that is, $D \xi(t)=Y^{0}(t, \xi(t))$ and $D_{*} \xi(t)=Y_{*}^{0}(t, \xi(t))$. 
Definition $2.2([10])$. A derivative $D_{S}=\frac{1}{2}\left(D+D_{*}\right)$ is called a symmetric mean derivative. The derivative $D_{A}=\frac{1}{2}\left(D-D_{*}\right)$ is called antisymmetric mean derivative.

We consider vector fields

$$
v^{\xi}(t, x)=\frac{1}{2}\left(Y^{0}(t, x)+Y_{*}^{0}(t, x)\right), \quad u^{\xi}(t, x)=\frac{1}{2}\left(Y^{0}(t, x)-Y_{*}^{0}(t, x)\right) .
$$

Definition $2.3([10])$. The quantity $v^{\xi}(t)=v^{\xi}(t, \xi(t))=D_{S} \xi(t)$ is called a current velocity of a process $\xi(t)$ and $u^{\xi}(t)=u^{\xi}(t, \xi(t))=D_{A} \xi(t)$ is called an osmotic velocity of process $\xi(t)$.

The current velocity for random process is a direct analogue of a usual physical velocity of deterministic processes, see [10]. The osmotic velocity measures how fast the randomness of the process increases.

A determinative role in our construction is played by the Wiener process [10, which we denote by the symbol $w(t)$. The following lemmata hold.

Lemma 2.1 ([16]). Let $w(t)$ be an $n$-dimensional Wiener process, $P(t)$ be a sufficiently smooth $k \times n$-matrix and $t \in(0, T)$. Then the formula

$$
D_{S}^{w} \int_{0}^{t} P(s) d w(s)=P(t) \frac{w(t)}{2 t}
$$

holds for each $t$.

Lemma 2.2 ([10], [11]). For $t \in(0, T)$, the identities

$$
D w(t)=0, \quad D_{*} w(t)=\frac{w(t)}{t}, \quad D_{S} w(t)=\frac{w(t)}{2 t}
$$

hold. For an integer $k \geqslant 2$, the identity

$$
D_{S}^{k} w(t)=(-1)^{k-1} \frac{\prod_{i=1}^{k-1}(2 i-1)}{2^{k}} \frac{w(t)}{t^{k}}
$$

is satisfied.

\section{MAIN RESULT}

As it has been said in the Introduction, we consider the stochastic differential equation in $\mathbb{R}^{n}$ of form

$$
d \tilde{A} \xi(t)=\tilde{B} \xi(t) d t+f(t) d t+d S \zeta(t)+P(t) d w(t), \quad 0 \leqslant t \leqslant T,
$$

which is in an integral form reads as

$$
\tilde{A} \xi(t)=\tilde{B} \int_{0}^{t} \xi(s) d s+\int_{0}^{t} f(s) d s+S \zeta(t)+\int_{0}^{t} P(s) d w(s), \quad 0 \leqslant t \leqslant T,
$$

where $\tilde{A}$ and $\tilde{B}$ are real constant matrices of size $n \times n$, the matrix $\tilde{A}$ is degenerate, $\tilde{B}+\lambda \tilde{A}$ is a regular matrix pencil, $\lambda \in \mathbb{R}, S$ is a scalar matrix of size $n \times n, \zeta(t)$ is an $n$-dimensional jump process, $P(t)$ is a sufficiently smooth matrix of size $n \times n, f(t)$ is a sufficiently smooth deterministic vector function depending on time, $w(t)$ is a Wiener process, $\xi(t)$ is a sought random process.

The jump process $\zeta(t)=\zeta(t, \omega)$ is defined as follows

$$
\zeta(t, \omega)=\sum_{r=1}^{N} \tilde{\zeta}_{r}(\omega) \chi\left(t-t_{r}\right), \quad 0<t_{1}<\cdots<t_{N}<T
$$


where $\chi$ is the Heaviside function vanishing for negative values of its variable and equalling to one for non-negative values, $\tilde{\zeta}_{r}(\omega)$ are random variables with values in $\mathbb{R}^{n}$.

In view of (3.1) it is clear that, for simplicity, an initial condition for solution of (3.1) is supposed to be

$$
\xi(0, \omega)=0 .
$$

We say from the very beginning that the solutions we construct do not satisfy this condition. This is why we approximate the solutions by processes satisfying this initial conditions but becoming solutions only from some, prescribed and arbitrary small, time $t_{0}>0$.

As in work [2], we seek solutions for problems (3.1), (3.2) among random processes $\xi(t, \omega)$ satisfying the equations

$$
\begin{aligned}
& \tilde{A} \xi(t)-\tilde{A} \xi(0)=\tilde{B} \int_{0}^{t} \xi(s) d s+\int_{0}^{t} f(s) d s+\int_{0}^{t} P(s) d w(s), \quad 0 \leqslant t \leqslant t_{1}, \\
& \tilde{A} \xi(t)-\tilde{A} \xi\left(t_{r}\right)=\tilde{B} \int_{t_{r}}^{t} \xi(s) d s+\int_{t_{r}}^{t} f(s) d s+\int_{t_{r}}^{t} P(s) d w(s), \quad t_{r} \leqslant t \leqslant t_{r+1}, \\
& \tilde{A} \xi(t)-\tilde{A} \xi\left(t_{N}\right)=\tilde{B} \int_{t_{N}}^{t} \xi(s) d s+\int_{t_{N}}^{t} f(s) d s+\int_{t_{N}}^{t} P(s) d w(s), \quad t_{N} \leqslant t \leqslant T,
\end{aligned}
$$

in the sense we describe below for all $r=1,2, \ldots, N-1$, satisfying the identities

$$
\tilde{A} \xi\left(t_{r}+0, \omega\right)-\tilde{A} \xi\left(t_{r}-0, \omega\right)=S \tilde{\zeta}_{r}(\omega), r=1,2, \ldots, N
$$

and satisfying initial condition $(3.2)$ at the initial time $t=0$.

Thus, the process $\xi(t)$ for solution of problem (3.1), (3.2) is determined successively for $r=0,1, \ldots, N$ via random processes $\xi_{r}(t)$ satisfying the equations

$$
\begin{array}{ll}
\tilde{A} \xi_{0}(t)-\tilde{A} \xi_{0}(0)=\tilde{B} \int_{0}^{t} \xi_{0}(s) d s+\int_{0}^{t} f(s) d s+\int_{0}^{t} P(s) d w(s), & 0 \leqslant t \leqslant t_{1}, \quad r=0, \\
\tilde{A} \xi_{r}(t)-\tilde{A} \xi_{r}\left(t_{r}\right)=\tilde{B} \int_{t_{r}}^{t} \xi_{r}(s) d s+\int_{t_{r}}^{t} f(s) d s+\int_{t_{r}}^{t} P(s) d w(s), & t_{r} \leqslant t \leqslant t_{r+1}, \\
\tilde{A} \xi_{N}(t)-\tilde{A} \xi_{N}\left(t_{N}\right)=\tilde{B} \int_{t_{N}}^{t} \xi_{N}(s) d s+\int_{t_{N}}^{t} f(s) d s+\int_{t_{N}}^{t} P(s) d w(s), & t_{N} \leqslant t \leqslant T,
\end{array}
$$

$r=1,2, \ldots, N-1$, where

$$
\xi_{0}(0)=0, \quad \tilde{A} \xi_{r}\left(t_{r}\right)=\tilde{A} \xi_{r-1}\left(t_{r}, \omega\right)+S \tilde{\zeta}_{r}(\omega), \quad r=1, \ldots, N
$$

In what follows we shall make use of the following theorem.

Theorem 3.1 (Generalized real Schur form 9]). Given a regular pencil $\lambda A+B$ of real constant matrices $A$ and $B$ of size $n \times n$, there exist real orthogonal matrices $Q_{L}$ and $Q_{R}$ such that the matrix $Q_{L} A Q_{R}$ is upper quasi-triangle, that is, an upper block-triangle matrix with diagonal blocks of size $1 \times 1$ and $2 \times 2$, the blocks of size $1 \times 1$ correspond to real eigenvalues and the blocks of size $2 \times 2$ do to conjugate pairs of complex eigenvalues. The matrix $Q_{L} B Q_{R}$ is upper triangle. 
It is easy to see that equation (3.1) in general form is inconvenient for studying and this is why we reduce it to some canonical form. For the regular matrix pencil $\lambda \tilde{A}+\tilde{B}, \lambda \in \mathbb{R}$ we make Shur transform, which is described by a pair of non-degenerate matrices $Q_{L}$ and $Q_{R}$. Then equation (3.1) is transformed as follows:

$$
\begin{aligned}
Q_{L} \tilde{A} Q_{R} Q_{\mathbb{R}}^{-1} \xi(t)= & Q_{L} \tilde{B} Q_{R} \int_{0}^{t} Q_{\mathbb{R}}^{-1} \xi(s) d s+\int_{0}^{t} Q_{L} f(s) d s \\
& +Q_{L} S \zeta(t)+\int_{0}^{t} Q_{L} P(s) d w(s), \quad 0 \leqslant t \leqslant T,
\end{aligned}
$$

and in new notations it becomes

$$
\begin{gathered}
A \eta(t)=\int_{0}^{t} B \eta(s) d s+\int_{0}^{t} g(s) d s+M \zeta(t)+\int_{0}^{t} C(s) d w(s), \\
\eta(0)=0,
\end{gathered}
$$

where $C(t)=Q_{L} P(t), \eta(t)=Q_{\mathbb{R}}^{-1} \xi(t), M=Q_{L} S, A=Q_{L} \tilde{A} Q_{R}$ is an upper quasi-triangle matrix, $B=Q_{L} \tilde{B} Q_{R}$ is an upper-triangle matrix, $Q_{L} f(t)=g(t)$. Indexing appropriately the vectors in a basis, in $A$, along the main diagonal, first the blocks of size $2 \times 2$ are located, then there are non-degenerate blocks of size $1 \times 1$, and then degenerate blocks of size $1 \times 1$ follow.

Then, in view of the said above, the formulae for solutions $\eta(t)$ to problem (3.3), (3.4) are determined successively for $r=0,1, \ldots, N$ via random processes $\eta_{r}(t)$ satisfying the equations

$$
\begin{gathered}
A \eta_{0}(t)-A \eta_{0}(0)=\int_{0}^{t} B \eta_{0}(s) d s+\int_{0}^{t} g(s) d s+\int_{0}^{t} C(s) d w(s), \quad 0 \leqslant t \leqslant t_{1}, \\
A \eta_{r}(t)-A \eta_{r}\left(t_{r}\right)=\int_{t_{r}}^{t} B \eta_{r}(s) d s+\int_{t_{r}}^{t} g(s) d s+\int_{t_{r}}^{t} C(s) d w(s), \\
t_{r} \leqslant t \leqslant t_{r+1}, \quad r=1, \ldots, N-1, \\
A \eta_{N}(t)-A \eta_{N}\left(t_{N}\right)=\int_{t_{N}}^{t} B \eta_{N}(s) d s+\int_{t_{N}}^{t} g(s) d s+\int_{t_{N}}^{t} C(s) d w(s), \quad t_{N} \leqslant t \leqslant T, \\
\eta_{0}(0)=0, \quad A \eta_{r}\left(t_{r}\right)=A \eta_{r-1}\left(t_{r}, \omega\right)+M \tilde{\zeta}_{r}(\omega), \quad r=1, \ldots, N .
\end{gathered}
$$

Remark 3.1. As it was mentioned above, to construct a process describing the model defined by equations (3.5), (3.6) and (3.7), we need the derivatives of free terms including the Wiener process. The derivatives of Wiener process exist only in the sense of generalized functions. This is why to avoid usage of generalized functions, to construct the process describing model defined by (3.5), (3.6) and (3.7), we shall employ symmetric mean derivatives (current velocities) $D_{S}^{w}$ for random processes. In this work, to calculate symmetric higher order derivatives, we shall employ the $\sigma$-algebra of "true" Wiener process. We note that for calculating mean derivatives one can use another $\sigma$-algebra, but this will change the formulae for calculating symmetric higher order derivatives of the Wiener process.

Taking into consideration the structure of matrices $A$ and $B$, it is easy to see that problems (3.3), (3.4) and (3.5), (3.6), (3.7), (3.8) split into several equations and systems of equations. 
We collect the last $n-p+1$ components of the process $\eta_{r}$ corresponding to the rows in $A$ with degenerate diagonal blocks of size $1 \times 1$ into a single matrix equation:

$$
\begin{aligned}
\left(\begin{array}{ccccc}
0 & a_{p+1}^{p} & a_{p+2}^{p} & \ldots & a_{n}^{p} \\
0 & 0 & a_{p+2}^{p+1} & \ldots & a_{n}^{p+1} \\
\vdots & \vdots & \vdots & \ddots & \vdots \\
0 & 0 & 0 & \ldots & 0
\end{array}\right)\left(\begin{array}{c}
\eta_{r}^{p}(t) \\
\eta_{r}^{p+1}(t) \\
\vdots \\
\eta_{r}^{n}(t)
\end{array}\right)-\left(\begin{array}{ccccc}
0 & a_{p+1}^{p} & a_{p+2}^{p} & \ldots & a_{n}^{p} \\
0 & 0 & a_{p+2}^{p+1} & \ldots & a_{n}^{p+1} \\
\vdots & \vdots & \vdots & \ddots & \vdots \\
0 & 0 & 0 & \ldots & 0
\end{array}\right)\left(\begin{array}{c}
\eta_{r}^{p}\left(t_{r}\right) \\
\eta_{r}^{p+1}\left(t_{r}\right) \\
\vdots \\
\eta_{r}^{n}\left(t_{r}\right)
\end{array}\right) \\
=\int_{t_{r}}^{t}\left(\begin{array}{cccc}
b_{p}^{p} & b_{p+1}^{p} & \ldots & b_{n}^{p} \\
0 & b_{p+1}^{p+1} & \ldots & b_{n}^{p+1} \\
\vdots & \vdots & \ldots & \vdots \\
0 & 0 & \ldots & b_{n}^{n}
\end{array}\right)
\end{aligned}
$$

By the last equation in system (3.9) we obtain that

$$
b_{n}^{n} \int_{t_{r}}^{t} \eta_{r}^{n}(s) d s=-\int_{t_{r}}^{t} g^{n}(s) d s-\sum_{j=1}^{n} \int_{t_{r}}^{t} c_{j}^{n}(s) d w^{j}(s)
$$

Since exactly the current velocity (symmetric mean derivative) corresponds to the physical velocity, from this equation we find $\eta_{r}^{n}(t)$ by applying the derivative $D_{S}^{w}$ to both sides of the identity, see Remark 3.1. It is easy to see that applying the mean derivatives $D^{w}$ and $D_{*}^{w}$, and, therefore, $D_{S}^{w}$, to the Riemann integrals in the left and right hand sides give the same results $\eta_{r}^{n}(t)$ and $g^{n}(t)$. Thus, by applying Lemma 2.1 we obtain that

$$
\eta_{r}^{n}(t)=-\frac{1}{b_{n}^{n}} g^{n}(t)-\frac{1}{b_{n}^{n}} \sum_{j=1}^{n} c_{j}^{n}(t) \frac{w^{j}}{2 t}, \quad r=1,2, \ldots, N-1 .
$$

By the pre-last equation in system $(3.9)$ we obtain that

$$
\begin{aligned}
a_{n}^{n-1} \eta_{r}^{n}(t)-a_{n}^{n-1} \eta_{r}^{n}\left(t_{r}\right)= & \int_{t_{r}}^{t}\left(b_{n-1}^{n-1} \eta^{n-1}(s)+b_{n}^{n-1} \eta^{n}(s)\right) d s \\
& +\int_{t_{r}}^{t} g^{n-1}(s) d s+\sum_{j=1}^{n} \int_{t_{r}}^{t} c_{j}^{n-1}(s) d w^{j}(s) .
\end{aligned}
$$

Arguing now as above and employing Lemma 2.2, we find that

$$
\begin{aligned}
\eta_{r}^{n-1}(t)= & -\frac{a_{n}^{n-1}}{b_{n-1}^{n-1} \cdot b_{n}^{n}} \cdot \frac{d g^{n}(t)}{d t}+\frac{b_{n}^{n-1}}{b_{n-1}^{n-1} \cdot b_{n}^{n}} \cdot g^{n}(t)-\frac{1}{b_{n-1}^{n-1}} \cdot g^{n-1}(t) \\
& +\frac{a_{n}^{n-1}}{b_{n-1}^{n-1} \cdot b_{n}^{n}} \cdot \sum_{j=1}^{n} c_{j}^{n}(t) \cdot \frac{w^{j}}{4 t^{2}}-\frac{a_{n}^{n-1}}{b_{n-1}^{n-1} \cdot b_{n}^{n}} \cdot \sum_{j=1}^{n} \frac{w^{j}}{2 t} \cdot \frac{d c_{j}^{n}(t)}{d t} \\
& +\frac{b_{n}^{n-1}}{b_{n-1}^{n-1} \cdot b_{n}^{n}} \cdot \sum_{j=1}^{n} c_{j}^{n}(t) \cdot \frac{w^{j}}{2 t}-\frac{1}{b_{n-1}^{n-1}} \cdot \sum_{j=1}^{n} c_{j}^{n-1}(t) \cdot \frac{w^{j}}{2 t}
\end{aligned}
$$


Exactly in the same way, for $p \leqslant i \leqslant n-1$ we obtain a recurrent formula:

$$
D_{S}\left(\sum_{j=i+1}^{n} a_{j}^{i} \cdot \eta_{r}^{j}(t)\right)=\sum_{j=i}^{n} b_{j}^{i} \cdot \eta_{r}^{j}(t)+g^{i}(t)+\sum_{j=1}^{n} c_{j}^{i}(t) \cdot \frac{w^{j}}{2 t} .
$$

We note that for systems of form $(3.9)$ defined on segments $\left[0, t_{1}\right]$ and $\left[t_{N}, T\right]$, similar formulae for solutions hold for $0<t \leqslant t_{1}$ and $t_{N} \leqslant t<T$. And the found processes satisfy conditions (3.8) if the components of the random variable $M \tilde{\zeta}_{r}(\omega)$ corresponding to zero $1 \times 1$ blocks in the main diagonal in $A$ vanish, that is, $\left(\left(M \tilde{\zeta}_{r}(\omega)\right)^{j}\right)_{j=p}^{n}=0$.

Thus, in view of the said above, as $0<t<T$, we obtain formulae for $\eta^{i}(t)$ :

$$
\begin{gathered}
\eta^{n}(t)=-\frac{1}{b_{n}^{n}} g^{n}(t)-\frac{1}{b_{n}^{n}} \sum_{j=1}^{n} c_{j}^{n}(t) \frac{w^{j}}{2 t}, \\
D_{S}\left(\sum_{j=i+1}^{n} a_{j}^{i} \cdot \eta^{j}(t)\right)=\sum_{j=i}^{n} b_{j}^{i} \cdot \eta^{j}(t)+g^{i}(t)+\sum_{j=1}^{n} c_{j}^{i}(t) \cdot \frac{w^{j}}{2 t}, \quad p \leqslant i \leqslant n-1 .
\end{gathered}
$$

We proceed to the issue on zero initial conditions for solutions of system $(3.9)$ as $r=0$. Taking into consideration the definition of mean symmetric derivatives it is easy to see that they are well-defined only on open time intervals since their constructions involve both increments to the left and to the right. Then by formulae (3.10), 3.11) and (3.12) we see that the solutions $\eta^{l}(t)$ are described as sums in which each terms involves a factor of form $\frac{w^{j}(t)}{t^{k}}, k \geqslant 1$. Therefore, the solutions tend to infinite as $t \rightarrow 0$, that is, the values of solutions do not exist at $t=0$. As in [11, one of the ways of resolving this situation is as follows. We fix an arbitrary small time $t_{0} \in(0, T)$ and defined a function $t_{0}(t)$ by the formula

$$
t_{0}(t)=\left\{\begin{array}{lll}
t_{0} & \text { if } & 0 \leqslant t \leqslant t_{0} \\
t & \text { if } & t_{0} \leqslant t
\end{array}\right.
$$

We replace the elements $\frac{w^{j}(t)}{t^{k}}$ in formulae 3.10, 3.11) and 3.12 by $\frac{w^{j}(t)}{\left(t_{0}(t)\right)^{k}}$. The obtained process vanish at the time $t=0$, but they become solutions only for $t_{0} \leqslant t<T$. We note that for two different times $t_{0}^{(1)}$ and $t_{0}^{(2)}$ with $t \geqslant \max \left(t_{0}^{(1)}, t_{0}^{(2)}\right)$ the values of the corresponding processes coincide almost surely.

For the rows of $A$ with non-degenerate blocks of size $1 \times 1$ we obtain the equations

$$
\begin{aligned}
a_{j}^{j} \eta_{r}^{j}(t) & +a_{j+1}^{j} \eta^{j+1}(t)+\ldots+a_{n}^{j} \eta^{n}(t)-a_{j}^{j} \eta^{j}\left(t_{r}\right)-a_{j+1}^{j} \eta^{j+1}\left(t_{r}\right)-\ldots-a_{n}^{j} \eta^{n}\left(t_{r}\right) \\
= & \int_{t_{r}}^{t}\left(b_{j}^{j} \eta_{r}^{j}(s)+b_{j+1}^{j} \eta^{j+1}(s)+\ldots+b_{n}^{j} \eta^{n}(s)\right) d s+\int_{t_{r}}^{t} g^{j}(s) d s+\int_{t_{r}}^{t} c_{1}^{j}(s) d w^{1}(s) \\
& +\int_{t_{r}}^{t} c_{2}^{j}(s) d w^{2}(s)+\cdots+\int_{t_{r}}^{t} c_{n}^{j}(s) d w^{n}(s), \quad t_{r} \leqslant t \leqslant t_{r+1}, \quad r=1, \ldots, N-1 .
\end{aligned}
$$


There exists an analytic formula for solutions of such equations, see [17]:

$$
\begin{aligned}
\eta_{r}^{j}(t)= & e^{\frac{b_{j}^{j}}{a_{j}^{j}}\left(t-t_{r}\right)} \eta_{r}^{j}\left(t_{r}\right)+\frac{a_{j+1}^{j}}{a_{j}^{j}} e^{\frac{b_{j}^{j}}{a_{j}^{j}}\left(t-t_{r}\right)} \eta^{j+1}\left(t_{r}\right)+\cdots+\frac{a_{n}^{j}}{a_{j}^{j}} e^{\frac{b_{j}^{j}}{a_{j}^{j}}\left(t-t_{r}\right)} \eta^{n}\left(t_{r}\right)+\int_{t_{r}}^{t} e^{\frac{b_{j}^{j}}{a_{j}^{j}}(t-u)} \frac{c_{1}^{j}(u)}{a_{j}^{j}} d w_{u}^{1} \\
& +\int_{t_{r}}^{t} e^{\frac{b_{j}^{j}}{a_{j}^{j}}(t-u)} \frac{c_{2}^{j}(u)}{a_{j}^{j}} d w_{u}^{2}+\cdots+\int_{t_{r}}^{t} e^{\frac{b_{j}^{j}}{a_{j}^{j}}(t-u)} \frac{c_{n}^{j}(u)}{a_{j}^{j}} d w_{u}^{n}+\int_{t_{r}} e^{\frac{b_{j}^{j}}{a_{j}^{j}}(t-u)}\left[\frac{1}{a_{j}^{j}} g^{j}(u)+\frac{b_{j+1}^{j}}{a_{j}^{j}} \eta^{j+1}(u)\right. \\
& \left.+\ldots+\frac{b_{n}^{j}}{a_{j}^{j}} \eta^{n}(u)-\frac{b_{j}^{j}}{\left(a_{j}^{j}\right)^{2}}\left(a_{j+1}^{j} \eta^{j+1}(u)+\ldots+a_{n}^{j} \eta^{n}(u)\right)\right] d u-\frac{a_{j+1}^{j}}{a_{j}^{j}} \eta^{j+1}-\ldots-\frac{a_{n}^{j}}{a_{j}^{j}} \eta^{n} .
\end{aligned}
$$

We note that for equations of form (3.16) defined on segments $\left[0, t_{1}\right]$ and $\left[t_{N}, T\right]$, similar formulae hold for their solutions. Taking into consideration all $\eta_{r}^{j}(t)$, we obtain the expressions for $\eta^{j}(t)$ :

$$
\begin{aligned}
\eta^{j}(t)= & \sum_{r=1}^{N} e^{\frac{b_{j}^{j}}{a_{j}^{j}}\left(t-t_{r}\right)} \cdot\left(\frac{m_{1}^{j} \tilde{\zeta}_{r}^{1}}{a_{j}^{j}}+\cdots+\frac{m_{n}^{j} \tilde{\zeta}_{r}^{n}}{a_{j}^{j}}\right) \cdot \chi\left(t-t_{r}\right)+\int_{0}^{t} e^{\frac{b_{j}^{j}}{a_{j}^{j}}(t-u)} \frac{c_{1}^{j}(u)}{a_{j}^{j}} d w_{u}^{1} \\
& +\int_{0}^{t} e^{\frac{b_{j}^{j}}{a_{j}^{j}}(t-u)} \frac{c_{2}^{j}(u)}{a_{j}^{j}} d w_{u}^{2}+\cdots+\int_{0}^{t} e^{\frac{b_{j}^{j}}{a_{j}^{j}}(t-u)} \frac{c_{n}^{j}(u)}{a_{j}^{j}} d w_{u}^{n}+\int_{0}^{\frac{b_{j}^{j}}{a_{j}^{j}}(t-u)}\left[\frac{1}{a_{j}^{j}} g^{j}(u)\right. \\
& \left.+\frac{b_{j+1}^{j}}{a_{j}^{j}} \eta^{j+1}(u)+\ldots+\frac{b_{n}^{j}}{a_{j}^{j}} \eta^{n}(u)-\frac{b_{j}^{j}}{\left(a_{j}^{j}\right)^{2}}\left(a_{j+1}^{j} \eta^{j+1}(u)+\ldots+a_{n}^{j} \eta^{n}(u)\right)\right] d u \\
& -\frac{a_{j+1}^{j}}{a_{j}^{j}} \eta^{j+1}-\ldots-\frac{a_{n}^{j}}{a_{j}^{j}} \eta^{n} .
\end{aligned}
$$

For the rows of $A$ with blocks of size $2 \times 2$ we obtain subsystems of the pairs of equations:

$$
\begin{array}{r}
a_{i}^{i} \eta_{r}^{i}(t)+a_{i+1}^{i} \eta_{r}^{i+1}(t)+a_{i+2}^{i} \eta^{i+2}(t)+\ldots+a_{n}^{i} \eta^{n}(t)-a_{i}^{i} \eta_{r}^{i}\left(t_{r}\right)-a_{i+1}^{i} \eta_{r}^{i+1}\left(t_{r}\right)-a_{i+2}^{i} \eta^{i+2}\left(t_{r}\right) \\
-\ldots-a_{n}^{i} \eta^{n}\left(t_{r}\right)=\int_{t_{r}}^{t}\left(b_{i}^{i} \eta_{r}^{i}(s)+b_{i+1}^{i} \eta_{r}^{i+1}(s)+b_{i+2}^{i} \eta^{i+2}(s)+\ldots+b_{n}^{i} \eta^{n}(s)\right) d s \\
\quad+\int_{t_{r}}^{t} g^{i}(s) d s+\int_{t_{r}}^{t} c_{1}^{i}(s) d w^{1}(s)+\int_{t_{r}}^{t} c_{2}^{i}(s) d w^{2}(s)+\ldots+\int_{t_{r}}^{t} c_{n}^{i}(s) d w^{n}(s), \\
a_{i}^{i+1} \eta_{r}^{i}(t)+a_{i+1}^{i+1} \eta_{r}^{i+1}(t)+a_{i+2}^{i+1} \eta^{i+2}(t)+\ldots+a_{n}^{i+1} \eta^{n}(t)-a_{i}^{i+1} \eta_{r}^{i}\left(t_{r}\right)-a_{i+1}^{i+1} \eta_{r}^{i+1}\left(t_{r}\right) \\
-a_{i+2}^{i+1} \eta^{i+2}\left(t_{r}\right)-\ldots-a_{n}^{i+1} \eta^{n}\left(t_{r}\right)=\int_{t_{r}}^{t}\left(b_{i+1}^{i+1} \eta_{r}^{i+1}(s)+b_{i+2}^{i+1} \eta^{i+2}(s)+\ldots+b_{n}^{i+1} \eta^{n}(s)\right) d s \\
+\int_{t_{r}}^{t} g^{i+1}(s) d s+\int_{t_{r}}^{t} c_{1}^{i+1}(s) d w^{1}(s)+\int_{t_{r}}^{t} c_{2}^{i+1}(s) d w^{2}(s)+\ldots+\int_{t_{r}}^{t} c_{n}^{i+1}(s) d w^{n}(s),
\end{array}
$$

$t_{r} \leqslant t \leqslant t_{r+1}, \quad r=1, \ldots, N-1$.

In a matrix form, in terms of new notations, this subsystem of equations becomes

$$
\bar{\eta}_{r}(t)-\bar{\eta}_{r}\left(t_{r}\right)+\vartheta(t)-\vartheta\left(t_{r}\right)=\int_{t_{r}}^{t} K \bar{\eta}_{r}(s) d s+\int_{t_{r}}^{t} \theta(s) d s+\int_{t_{r}}^{t} \bar{g}(s) d s+\int_{t_{r}}^{t} \Lambda \Theta(s) d w(s)
$$


where

$$
\begin{aligned}
& \bar{\eta}_{r}=\left(\begin{array}{c}
\eta_{r}^{i} \\
\eta_{r}^{i+1}
\end{array}\right), \quad \Xi=\left(\begin{array}{cc}
b_{i}^{i} & b_{i+1}^{i} \\
0 & b_{i+1}^{i+1}
\end{array}\right), \quad \Lambda=\left(\begin{array}{cc}
a_{i}^{i} & a_{i+1}^{i} \\
a_{i}^{i+1} & a_{i+1}^{i+1}
\end{array}\right)^{-1}, \\
& \vartheta(t)=\Lambda\left(\begin{array}{ccc}
a_{i+2}^{i} & \cdots & a_{n}^{i} \\
a_{i+2}^{i+1} & \cdots & a_{n}^{i+1}
\end{array}\right)\left(\eta^{i+2} \ldots \eta^{n}\right)^{T}, \quad \theta(t)=\Lambda\left(\begin{array}{ccc}
b_{i+2}^{i} & \cdots & b_{n}^{i} \\
b_{i+2}^{i+1} & \cdots & b_{n}^{i+1}
\end{array}\right)\left(\eta^{i+2} \ldots \eta^{n}\right)^{T}, \\
& \bar{g}=\Lambda\left(\begin{array}{c}
g^{i} \\
g^{i+1}
\end{array}\right), \quad K=\Lambda \Xi, \quad \Theta(t)=\left(\begin{array}{ccc}
c_{1}^{i}(t) & \cdots & c_{n}^{i}(t) \\
c_{1}^{i+1}(t) & \cdots & c_{n}^{i+1}(t)
\end{array}\right)
\end{aligned}
$$

For this subsystem of equations, the solutions can be found analytically, see [17]:

$$
\begin{aligned}
\bar{\eta}_{r}(t)= & e^{K\left(t-t_{r}\right)} \bar{\eta}_{r}\left(t_{r}\right)+e^{K\left(t-t_{r}\right)} \vartheta\left(t_{r}\right)+\int_{t_{r}}^{t} e^{K(t-\tau)} \Lambda \Theta(\tau) d w_{\tau} \\
& +\int_{t_{r}}^{t} e^{K(t-\tau)}(\theta(\tau)+\bar{g}(\tau)-K \vartheta(\tau)) d \tau-\vartheta(t) .
\end{aligned}
$$

We note that similar formulae hold for solutions to equations of form 3.18 defined on segments $\left[0, t_{1}\right]$ and $\left[t_{N}, T\right]$. Taking into consideration all $\bar{\eta}_{r}(t)$, we obtain an expression for $\bar{\eta}(t)$ :

$$
\begin{aligned}
\bar{\eta}(t)= & \sum_{r=1}^{N} e^{K\left(t-t_{r}\right)} \Lambda\left(\left(M \tilde{\zeta}_{r}(\omega)\right)^{j}\right)_{j=i}^{i+1} \cdot \chi\left(t-t_{r}\right)+\int_{0}^{t} e^{K(t-\tau)}(\theta(\tau)+\bar{g}(\tau)-K \vartheta(\tau)) d \tau \\
& +\int_{0}^{t} e^{K(t-\tau)} \Lambda \Theta(\tau) d w(\tau)-\vartheta(t),
\end{aligned}
$$

where $\left(\left(M \tilde{\zeta}_{r}(\omega)\right)^{j}\right)_{j=i}^{i+1}$ is a 2-dimensional vector formed by the $i$ th and $(i+1)$ th coordinates of the vector $M \tilde{\zeta}_{r}(\omega)$.

Thus, summarizing the said above, we arrive at the following statement.

Theorem 3.2. Let $\lambda \tilde{A}+\tilde{B}$ be a regular pencil of constant matrices of size $n \times n$, $S$ be a matrix of size $n \times n$, and $f(t)$ be a sufficiently smooth $n$-dimensional vector function, $0 \leqslant t \leqslant T$, $0<t_{1}<\cdots<t_{N}<T, Q_{L}$ and $Q_{R}$ be non-degenerate matrices of size $n \times n$ reducing the pencil $\lambda \tilde{A}+\tilde{B}$ to a canonical generalized Shur form, $A=Q_{L} \tilde{A} Q_{R}, B=Q_{L} \tilde{B} Q_{R}$. Let $\tilde{\zeta}_{r}(\omega)$ be random variables taking values in $\mathbb{R}^{n}$ such that the components of the random variable $Q_{L} S \tilde{\zeta}_{r}(\omega)$ corresponding to degenerate blocks of size $1 \times 1$ on the main diagonal in $A$ vanish, $r=1,2, \ldots, N$. Let $\zeta(t, \omega)=\sum_{r=1}^{N} \tilde{\zeta}_{r}(\omega) \chi\left(t-t_{r}\right)$, where $\chi$ is the Heaviside function vanishing for negative values of its independent variable and equalling to one for positive values. Then

1) equation (3.1) is reduced to canonical equation (3.3), which splits into separate equations and subsystems of equations;

2) for the subsystem corresponding to the rows of $A$ with degenerate blocks of size $1 \times 1$, recurrent formula (3.13), (3.14) for solutions hold as $0<t<T$;

3) fixing arbitrary small time $t_{0}>0$, in the denominators of the processes satisfying recurrent relations given in Statement 2, we replace $t$ by $t_{0}(t)$ defined in (3.15) and we obtain the processes vanishing at $t=0$ but being solutions only as $t_{0} \leqslant t<T$;

4) the solutions of the equations corresponding to rows of $A$ with non-degenerate blocks of size $1 \times 1$ satisfy analytic formula (3.17); 
5) the solutions of subsystems corresponding to rows of $A$ with non-degenerate blocks of size $2 \times 2$ satisfy analytic formula (3.19).

\section{BIBLIOGRAPHY}

1. L.A. Vlasenko, Yu.G. Lysenko, A.G. Rutkas. About one stochastic model of enterprise corporations dynamics // Economic Cybernetics. 1-3 (67-69), 4-9 (2011). (in Russian).

2. Vlasenko L.A., Lyashko S.L., Rutkas A.G. On a stochastic impulsive sustem // Dopov. Nats. Akad. Nauk Ukr. Mat. Pryr. Tekh. Nauky. 2, 50-55 (2012). (in Russian).

3. O. Schein, G. Denk. Numerical solution of stochastic differential-algebraic equations with applications to transient noise simulation of microelectronic circuits // J. Comp. Appl. Math. 100:1, 77-92 (1998).

4. T. Sickenberger, R. Winkler. Stochastic oscillations in circuit simulation // Proc. Appl. Math. Mech. 7:1, 4050023-4050024 (2007)

5. R. Winkler. Stochastic DAEs in transient noise simulation // Proc. Scientific Computing in Electrical Engineering (2002), Eindhoven. Springer Series Mathematics in Industry. 4, 408-415 (2004).

6. A.L. Shestakov, G.A. Sviridyuk. On the measurement of the "white noise" // Vestnik YuUrGU. Ser. Mat. Model. Progr. 13, 99-108 (2012). (in Russian).

7. A.A. Belov, A.P. Kurdyukov A.P. Descriptor systems and control problems. Fizmatlit, Moscow (2015). (in Russian).

8. E.Yu. Mashkov. Stochastic Leontief type equations with impulse actions // Vestnik YuUrGU. Ser. Mat. Model. Progr. 11:2, 58-72 (2018). (in Russian).

9. J. Demmel. Applied numerical linear algebra. SIAM, Philadelphia (1997).

10. Yu.E. Gliklikh. Global and stochastic analysis with applications to mathematical physics. Springer, Berlin (2011).

11. Yu.E. Gliklikh, E.Yu. Mashkov. Stochastic Leontieff type equations and mean derivatives of stochastic processes // Vestnik YuUrGU. Ser. Mat. Model. Progr. 6:2, 25-39 (2013).

12. E. Nelson. Derivation of the Schrödinger equation from Newtonian mechanics // Phys. Reviews. 150:4, 1079-1085 (1996).

13. E. Nelson. Dynamical theory of Brownian motion. Princeton Univ. Press, Princeton (1967).

14. E. Nelson. Quantum fluctuations. Princeton Univ. Press, Princeton (1985).

15. K.R. Parthasaraty. Introduction to probability and measure. Macmillan India Ltd., New Delhi (1977).

16. Yu.E. Gliklikh, E.Yu. Mashkov. Stochastic Leontief type equation with non-constant coefficients // Appl. Anal. 94:8, 1614-1623 (2015).

17. I.I. Gihman, A.V. Scorohod. Theory of stochastic processes. III. Springer-Verlag, Berlin (1979).

Evgenii Yurievich Mashkov,

Southwest State University,

50 let Oktyabrya str. 94,

305040, Kursk, Russia

E-mail: mashkovevgen@yandex.ru 